\title{
A polynomial investigation inspired by work of Schinzel and Sierpiński
}

\author{
Michael Filaseta \\ Department of Mathematics \\ University of South Carolina \\ Columbia, SC 29208 \\ U.S.A. \\ E-mail: filaseta@math.sc.edu \\ URL: http://www.math.sc.edu/ filaseta/ \\ Joshua Harrington \\ Department of Mathematics \\ University of South Carolina \\ Columbia, SC 29208 \\ U.S.A. \\ E-mail: harrinjs@mailbox.sc.edu
}

With best wishes to Schinzel for his 75th birthday.

\section{Introduction}

Define a covering system (or covering) of the integers as a finite collection of congruences $x \equiv a_{j}\left(\bmod m_{j}\right)$, with $1 \leq j \leq r$, such that every integer satisfies at least one of these congruences. As an interesting application of coverings, W. Sierpiński [4] showed that there are odd positive integers $k$ for which $k \cdot 2^{n}+1$ is composite for all integers $n \geq 0$. For $d \in \mathbb{Z}$, the first author [1] considered the analogous problem of finding $f(x) \in \mathbb{Z}[x]$ such

2000 Mathematics Subject Classification: $11 \mathrm{C} 08$ (11A07, 11B25, 11R09)

Key Words: covering system, polynomial 
that $f(x) \cdot x^{n}+d$ is reducible over the rationals for all integers $n \geq 0$. To make the problem non-trivial, we also require here that $f(1) \neq-d$. This problem was motivated by the work of A. Schinzel in [3]. Among the open problems on covering systems is the problem of determining whether there is an odd covering, that is a covering that consists of distinct odd moduli $>1$. Schinzel showed that if there is an $f(x) \in \mathbb{Z}[x]$ such that $f(1) \neq-1$ and $f(x) \cdot x^{n}+1$ is reducible for all integers $n \geq 0$, then there must be an odd covering. In fact, he showed considerably more than this, and the reader is directed to [3] for more details. For the general problem concerning $f(x) x^{n}+d$, the following is an easy consequence of the work of Schinzel [3] (see also [1]).

Theorem 1.1. Let $d$ be an odd integer. If there is an $f(x) \in \mathbb{Z}[x]$ satisfying $f(1) \neq-d$ and $f(x) \cdot x^{n}+d$ is reducible over the rationals for all integers $n \geq 0$, then there is an odd covering of the integers.

The polynomial

$$
f(x)=5 x^{9}+6 x^{8}+3 x^{6}+8 x^{5}+9 x^{3}+6 x^{2}+8 x+3
$$

motivated by an example given by Schinzel in [3], satisfies $f(1) \neq-12$ and $f(x) x^{n}+12$ is reducible for all $n \geq 0$. To justify the latter, one can make use of the following implications:

$$
\begin{aligned}
& n \equiv 0(\bmod 2) \Longrightarrow f(x) x^{n}+12 \equiv 0(\bmod x+1) \\
& n \equiv 2(\bmod 3) \Longrightarrow f(x) x^{n}+12 \equiv 0 \quad\left(\bmod x^{2}+x+1\right) \\
& n \equiv 1(\bmod 4) \Longrightarrow f(x) x^{n}+12 \equiv 0\left(\bmod x^{2}+1\right) \\
& n \equiv 1(\bmod 6) \Longrightarrow f(x) x^{n}+12 \equiv 0\left(\bmod x^{2}-x+1\right) \\
& n \equiv 3(\bmod 12) \Longrightarrow f(x) x^{n}+12 \equiv 0\left(\bmod x^{4}-x^{2}+1\right) \text {. }
\end{aligned}
$$

The congruences involving $n$ on the left can be shown to form a covering of the integers; in other words, every integer $n$ will satisfy at least one of these congruences. Each implication can be justified by noting that the modulus on the right is a cyclotomic polynomial $\Phi_{m}(x)$ with $m$ corresponding to the modulus used on $n$ on the left. We deduce from these implications that for each integer $n \geq 0$, the polynomial $f(x) x^{n}+12$ is divisible by $\Phi_{m}(x)$ for some $m$ dividing 12 and, hence, reducible.

In [1], the first author showed that a similar example exists whenever $d$ is an integer divisible by 4 . Thus, if $4 \mid d$, then there is an $f(x) \in \mathbb{Z}[x]$ such that $f(1) \neq-d$ and $f(x) \cdot x^{n}+d$ is reducible over the rationals for all integers $n \geq 0$. L. Jones [2] has shown that there are also similar examples for infinitely many positive integers $d \equiv 2(\bmod 4)$. The smallest such $d$ he gives with his method is $d=90$.

The purpose of this paper is to improve on the work in [1] and [2] by showing that examples similar to Schinzel's example above exist for every even integer $d$. Thus, examples exist for every $d$ for which Theorem 1.1 does not apply. Specifically, we show the following. 
Theorem 1.2. Let $d$ be an even integer. There is an $f(x) \in \mathbb{Z}[x]$ satisfying both $f(1) \neq-d$ and $f(x) \cdot x^{n}+d$ is reducible over the rationals for all integers $n \geq 0$.

\section{Further Preliminaries}

Our arguments begin with the following lemma which appears in [1].

Lemma 2.1. Let $d$ be a positive integer. Suppose that $S$ is a system of congruences

$$
x \equiv 2^{j-1} \quad\left(\bmod 2^{j}\right) \quad \text { for } j \in\{1,2, \ldots, k\}
$$

for some positive integer $k$ together with

$$
x \equiv a_{j} \quad\left(\bmod m_{j}\right) \quad \text { for } j \in\{1,2, \ldots, r\}
$$

for some positive integer $r$ satisfying:

(i) The system $S$ is a covering of the integers.

(ii) The moduli in (2.1) and (2.2) are all distinct and $>1$.

(iii) For each $j \in\{1,2, \ldots, r\}$,

$$
\left(\prod_{\substack{1 \leq i \leq r \\ i \neq j}} a(i, j)\right)\left(\prod_{i=1}^{k} b(i, j)\right) \text { divides } d
$$

where

$$
a(i, j)= \begin{cases}p & \text { if } m_{i} / m_{j}=p^{t} \text { for some prime } p \text { and some integer } t \\ 1 & \text { otherwise }\end{cases}
$$

and

$$
b(i, j)= \begin{cases}p & \text { if } m_{j} / 2^{i}=p^{t} \text { for some prime } p \text { and some integer } t \\ 1 \quad \text { otherwise }\end{cases}
$$

(iv) The double product $\prod_{i=1}^{k} \prod_{j=1}^{r} b(i, j)$ divides $d$.

Then there exists $f(x) \in \mathbb{Z}[x]$ with positive coefficients such that $f(x) x^{n}+d$ is reducible over the rationals for all non-negative integers $n$.

We proceed now as follows. Next, we state a lemma establishing the existence of a certain covering system. Then we will explain how this lemma will allow us to obtain Theorem 1.2. Finally, we give a proof of the lemma by explicitly establishing the needed covering. 
Lemma 2.2. There is a covering of the integers consisting of moduli $m_{1}, m_{2}$, $\ldots, m_{r}$ satisfying:

(i) Each $m_{\ell}$ is odd and $>1$.

(ii) If $m_{\ell}$ is a prime number, then $m_{j} \neq m_{\ell}$ for each $j \neq \ell$, with $1 \leq j \leq r$.

(iii) If $m_{\ell}$ has at least two distinct prime factors, then there is at most one $j \neq \ell$, with $1 \leq j \leq r$, such that $m_{j}=m_{\ell}$.

We show that Lemma 2.2 implies that there is an $f(x) \in \mathbb{Z}[x]$ with positive coefficients such that $f(x) x^{n}+2$ is reducible over the rationals for all non-negative integers $n$. Observe that by simply multiplying through by an appropriate positive integer, we can deduce that for every even integer $d$, there is an $f(x) \in \mathbb{Z}[x]$, depending on $d$, with positive coefficients such that $f(x) x^{n}+d$ is reducible over the rationals for all non-negative integers $n$. More interesting examples, where for example the greatest common divisor of the coefficients of $f(x)$ is 1 , can be obtained for general even $d$ by adding a polynomial of the form $x^{m}+x^{m-1}+\cdots+x+1$ for an appropriate large positive integer $m$. In any case, $f(1) \neq-d$.

To obtain $f(x) \in \mathbb{Z}[x]$ with positive coefficients such that $f(x) x^{n}+2$ is reducible over the rationals for all non-negative integers $n$, Lemma 2.1 implies that we need only show the existence of a certain covering system. Our goal is to revise the covering system in Lemma 2.2 to show that the covering system for Lemma 2.1 exists.

Let $x \equiv a_{j}\left(\bmod m_{j}\right)$ for $j \in\{1,2, \ldots, r\}$ denote the $r$ congruences given by Lemma 2.2. Suppose that the first $s$ of these are primes and the remaining are not. We suppose as we may (from Lemma 2.2 (iii)) that if $m_{i}=m_{j}$ for some integers $i$ and $j$ with $s+1 \leq j<i \leq r$, then $i=j+1$. Define

$$
m_{j}^{\prime}= \begin{cases}m_{j} & \text { for } j \in\{1,2, \ldots, s\} \\ 2^{j-s} m_{j} & \text { for } j \in\{s+1, s+2, \ldots, r\} .\end{cases}
$$

Let $b_{j}=a_{j}$ for $1 \leq j \leq s$. For each $j \in\{s+1, s+2, \ldots, r\}$, we define $b_{j}$ as the nonnegative integer $<2^{j-s} m_{j}$ satisfying

$$
b_{j} \equiv a_{j}\left(\bmod m_{j}\right) \quad \text { and } \quad b_{j} \equiv 0\left(\bmod 2^{j-s}\right) \text {, }
$$

which exists by the Chinese Remainder Theorem. We consider the congruences

$$
x \equiv 2^{j-1}\left(\bmod 2^{j}\right) \quad \text { for } j \in\{1,2, \ldots, r-s\}
$$

together with

$$
x \equiv b_{j}\left(\bmod m_{j}^{\prime}\right) \quad \text { for } j \in\{1,2, \ldots, r\}
$$


We show that these congruences form a system $S$ of congruences satisfying the conditions of Lemma 2.1 with $d=2, k=r-s$ and the $m_{j}$ there replaced by $m_{j}^{\prime}$.

Suppose $n$ is an integer that does not satisfy one of the congruences in (2.3). Observe that if $j$ is the largest positive integer for which $2^{j-1}$ divides $n$, then $n \equiv 2^{j-1}\left(\bmod 2^{j}\right)$. Since $n$ does not satisfy the congruences in $(2.3)$, we deduce $n \equiv 0\left(\bmod 2^{r-s}\right)$. Also, since the congruences $x \equiv a_{j}\left(\bmod m_{j}\right)$ for $j \in\{1,2, \ldots, r\}$ form a covering of the integers, $n \equiv a_{j}\left(\bmod m_{j}\right)$ for some $j \in\{1,2, \ldots, r\}$. By the definition of $b_{j}$, we have for that choice of $j$ that $x \equiv b_{j}\left(\bmod m_{j}^{\prime}\right)$. Hence, $n$ satisfies one of the congruences in $(2.4)$. Thus, $S$ satisfies the condition (i) of Lemma 2.1 .

Condition (ii) of Lemma 2.1 is easily checked for the congruences in (2.3) and (2.4). To verify conditions (iii) and (iv) of Lemma 2.1 for the congruences in (2.3) and (2.4), we alter the definitions of $a(i, j)$ and $b(i, j)$ accordingly so that $m_{i}$ and $m_{j}$ are replaced by $m_{i}^{\prime}$ and $m_{j}^{\prime}$. Note that the conditions in Lemma 2.2 and the definition of $s$ imply that $m_{1}^{\prime}, m_{2}^{\prime}, \ldots, m_{s}^{\prime}$ are distinct primes and $m_{s+1}^{\prime}, m_{s+2}^{\prime}, \ldots, m_{r}^{\prime}$ are distinct numbers each having $\geq 3$ distinct prime factors. Further, the largest powers of 2 dividing the numbers $m_{s+1}^{\prime}, m_{s+2}^{\prime}, \ldots, m_{r}^{\prime}$, namely $2,2^{2}, \ldots, 2^{r-s}$, are distinct. It follows that if the ratio $m_{j}^{\prime} / m_{i}^{\prime}=p^{t}$ for some prime $p$ and some integer $t$, then $p=2$ and, consequently, $m_{j}=m_{i}$. Recall that for $j$ fixed, the conditions $i \neq j$ and $m_{j}=m_{i}$ imply there is at most one possibility for $i$. We deduce that for each $j \in\{1,2, \ldots, r\}$,

$$
\prod_{\substack{1 \leq i \leq r \\ i \neq j}} a(i, j) \text { divides } 2 \text {. }
$$

The conditions in Lemma 2.2 imply that each $m_{j}$ with $j>s$ and, hence, each even $m_{j}^{\prime}$ has at least two odd prime divisors. It follows that $b(i, j)=1$ for every choice of $i$ and $j$ in $\{1,2, \ldots, r\}$. Conditions (iii) and (iv) of Lemma 2.1 now easily follow.

\section{Construction for Lemma 2.2}

Given lists $\left[b_{1}, \ldots, b_{t}\right]$ and $\left[n_{1}, \ldots, n_{t}\right]$ with $n_{1}, \ldots, n_{t}$ pairwise relatively prime positive integers, we denote by

$$
\left(\left[b_{1}, \ldots, b_{t}\right],\left[n_{1}, \ldots, n_{t}\right]\right)
$$

the congruence $x \equiv b \bmod n$ where $n=n_{1} \cdots n_{t}$ and $b \in\{0,1, \ldots, n-1\}$ satisfies $b \equiv b_{j}\left(\bmod n_{j}\right)$ for $1 \leq j \leq t$. That such a $b$ exists follows from the Chinese Remainder Theorem. Note that, with $b$ and $n$ so defined, the congruences represented by $\left(\left[b_{1}, \ldots, b_{t}\right],\left[n_{1}, \ldots, n_{t}\right]\right)$ and $([b],[n])$ are identical. With this same notation, we denote by

$$
\mathcal{I}\left(\left[b_{1}, \ldots, b_{t}\right],\left[n_{1}, \ldots, n_{t}\right]\right)=\mathcal{I}([b],[n])
$$


the set of integers satisfying $x \equiv b \bmod n$. We say that a collection of congruences covers a set of integers if every integer in the set satisfies at least one congruence in the collection.

In this section, we elaborate on the covering system, say $\mathcal{S}$, satisfying the conditions of Lemma 2.2. Noting that every integer belongs to one of the sets $\mathcal{I}([1],[3]), \mathcal{I}([2],[3])$ and $\mathcal{I}([3],[3])=\mathcal{I}([0],[3])$, we determine congruences for $\mathcal{S}$ by finding collections $\mathcal{S}_{1}, \mathcal{S}_{2}$ and $\mathcal{S}_{3}$ of congruences that cover each of these three sets. The system $\mathcal{S}$, then, will be the union of the congruences given in $\mathcal{S}_{1}, \mathcal{S}_{2}$ and $\mathcal{S}_{3}$.

For $\mathcal{I}([1],[3])$, we simply use the congruence $([1],[3])$. For $\mathcal{I}([2],[3])$, we consider the integers in each of the five residue classes modulo 5 . We cover the integers in $\mathcal{I}([2],[3])$ that are 1 modulo 5 by using the congruence $([1],[5])$. For later purposes, we note that this same congruence will cover the integers in $\mathcal{I}([3],[3])$ that are 1 modulo 5 . We cover the integers in $\mathcal{I}([2],[3])$ that are 2 modulo 5 and 5 modulo 5 by using congruences modulo 15 . Recall that the conditions in Lemma 2.2 allow us to use the modulus 15 for two different congruences. Thus, we can use the two congruences $([2,2],[3,5])$ and $([2,5],[3,5])$. So far our congruences $\mathcal{S}$ include the four congruences given by

$$
([1],[3]), \quad([1],[5]), \quad([2,2],[3,5]), \quad([2,5],[3,5]) .
$$

With these, we have covered $\mathcal{I}([1],[3])$ and three-fifths of $\mathcal{I}([2],[3])$. We still need to elaborate on the congruences of $\mathcal{S}$ that cover the integers in $\mathcal{I}([2],[3])$ that are 3 and 4 modulo 5 and that cover the integers in $\mathcal{I}([3],[3])$ (that are not 1 modulo 5).

We explain next the congruences used to cover $\mathcal{I}([2,3],[3,5])$. We will make use here of moduli of the form $3^{j+1} \cdot 5$ and moduli of the form $3^{j} \cdot 5 \cdot 23$ where $j$ is a positive integer. We keep in mind that each such modulus can be used for two congruences in $\mathcal{S}$, though we only take advantage of this fact for those of the form $3^{j+1} \cdot 5$. Each integer in $\mathcal{I}([2],[3])$ is either 2,5 or 8 modulo 9 . In the first two of these three cases, the integers that are also 3 modulo 5 satisfy one of the congruences $\left([2,3],\left[3^{2}, 5\right]\right)$ and $\left([5,3],\left[3^{2}, 5\right]\right)$. The integers that are 8 modulo 9 are either 8,17 or 26 modulo 27. Those that are 8 or 17 modulo 27 satisfy one of the congruences $\left([8,3],\left[3^{3}, 5\right]\right)$ and $\left([17,3],\left[3^{3}, 5\right]\right)$. In general, for each positive integer $j$, the integers that are $3^{j}-1$ modulo $3^{j}$ are either $3^{j}-1,2 \cdot 3^{j}-1$ or $3 \cdot 3^{j}-1=3^{j+1}-1$ modulo $3^{j+1}$. Those that are $3^{j}-1$ or $2 \cdot 3^{j}-1$ modulo $3^{j+1}$ and 3 modulo 5 are covered by $\left(\left[3^{j}-1,3\right],\left[3^{j+1}, 5\right]\right)$ and $\left(\left[2 \cdot 3^{j}-1,3\right],\left[3^{j+1}, 5\right]\right)$. We deduce that the congruences

$$
\left(\left[3^{j}-1,3\right],\left[3^{j+1}, 5\right]\right), \quad\left(\left[2 \cdot 3^{j}-1,3\right],\left[3^{j+1}, 5\right]\right), \quad \text { for } 1 \leq j \leq 22,
$$

cover all the integers in $\mathcal{I}([2],[3])$ that are 3 modulo 5 except those that are $3^{23}-1$ modulo $3^{23}$. Since each such integer is also congruent to some positive integer $\leq 23$ modulo 23 , we deduce that these integers are covered 
by the congruences

$$
\left(\left[3^{23}-1,3, j\right],\left[3^{j}, 5,23\right]\right), \quad \text { for } 1 \leq j \leq 23 .
$$

Thus, the congruences in (3.2) and (3.3) cover the integers in $\mathcal{I}([2],[3])$ that are 3 modulo 5 .

To finish covering the integers that are in $\mathcal{I}([2],[3])$, we are left with finding congruences that cover those that are also 4 modulo 5 . In other words, we are wanting now to cover $\mathcal{I}([2,4],[3,5])$. We divide these integers into classes modulo 7 , covering each in turn. We start with the congruences

$$
([1],[7]), \quad([2,4,2],[3,5,7]), \quad([2,4,3],[3,5,7]), \quad([4,4],[5,7]),
$$

to cover those integers in $\mathcal{I}([2,4],[3,5])$ that are $1,2,3$ or 4 modulo 7 . Next, we mimic what was done in $(3.2)$ and $(3.3)$ to cover $\mathcal{I}([2,3],[3,5])$ by restricting these same congruences to integers that are 5 modulo 7 . Specifically, we include

$$
\left(\left[3^{j}-1,4,5\right],\left[3^{j+1}, 5,7\right]\right),\left(\left[2 \cdot 3^{j}-1,4,5\right],\left[3^{j+1}, 5,7\right]\right), \text { for } 1 \leq j \leq 22,
$$

and

$$
\left(\left[3^{23}-1,4,5, j\right],\left[3^{j}, 5,7,23\right]\right), \quad \text { for } 1 \leq j \leq 23,
$$

in our system $\mathcal{S}$ to cover $\mathcal{I}([2,4,5],[3,5,7])$. To finish covering $\mathcal{I}([2,4],[3,5])$, we consider separately those in $\mathcal{I}([2,4,6],[3,5,7])$ and $\mathcal{I}([2,4,7],[3,5,7])$.

To cover $\mathcal{I}([2,4,6],[3,5,7])$, we use that each is in one of 7 different residue classes modulo $7^{2}$. We keep in mind that, although our required system $\mathcal{S}$ for Lemma 2.2 can involve moduli divisible by $p^{e}$ where $p$ is an odd prime and $e$ an integer $\geq 2$, it cannot have moduli that are equal to these prime powers. For $\mathcal{I}([2,4,6],[3,5,7])$, we cover 6 of the needed residue classes modulo $7^{2}$ using

$$
\begin{array}{cl}
\left([2,6],\left[3,7^{2}\right]\right), \quad\left([2,13],\left[3,7^{2}\right]\right), & \left([4,20],\left[5,7^{2}\right]\right), \quad\left([4,27],\left[5,7^{2}\right]\right), \\
\left([2,4,34],\left[3,5,7^{2}\right]\right), & \left([2,4,41],\left[3,5,7^{2}\right]\right) .
\end{array}
$$

For the final residue class modulo $7^{2}$, we use congruences similar to (3.2) and (3.3). This last class modulo $7^{2}$ is covered by

$$
\begin{gathered}
\left(\left[3^{j}-1,4,48\right],\left[3^{j+1}, 5,7^{2}\right]\right), \\
\left(\left[2 \cdot 3^{j}-1,4,48\right],\left[3^{j+1}, 5,7^{2}\right]\right), \quad \text { for } 1 \leq j \leq 22, \\
\left(\left[3^{23}-1,4,48, j\right],\left[3^{j}, 5,7^{2}, 23\right]\right), \quad \text { for } 1 \leq j \leq 23 .
\end{gathered}
$$

To finish covering $\mathcal{I}([2],[3])$, we need only cover $\mathcal{I}([2,4,7],[3,5,7])$. This is a thin enough set that we are able to get away with using the prime 19 to complete this case. The idea then is to consider each of the 19 possible 
residue classes that each of these integers can belong to. We cover 15 of these residue classes using

$$
\begin{gathered}
([1],[19]), \quad([2,2],[3,19]), \quad([2,3],[3,19]), \quad([4,4],[5,19]), \\
([4,5],[5,19]), \quad([2,4,6],[3,5,19]), \quad([2,4,7],[3,5,19]), \\
([7,8],[7,19]), \quad([7,9],[7,19]), \quad([2,7,10],[3,7,19]), \\
([2,7,11],[3,7,19]), \quad([4,7,12],[5,7,19]), \quad([4,7,13],[5,7,19]), \\
([2,4,7,14],[3,5,7,19]), \quad([2,4,7,15],[3,5,7,19]) .
\end{gathered}
$$

We make use of the idea in (3.2) and (3.3) to cover the remaining classes modulo 19, each class making use of such a list of congruences. Those integers congruent to 16 modulo 19 in $\mathcal{I}([2,4,7],[3,5,7])$ are covered by

$$
\begin{gathered}
\left(\left[3^{j}-1,16\right],\left[3^{j+1}, 19\right]\right),\left(\left[2 \cdot 3^{j}-1,16\right],\left[3^{j+1}, 19\right]\right), \text { for } 1 \leq j \leq 22 \\
\left(\left[3^{23}-1,16, j\right],\left[3^{j}, 19,23\right]\right), \quad \text { for } 1 \leq j \leq 23
\end{gathered}
$$

those congruent to 17 modulo 19 by

$$
\begin{aligned}
& \left(\left[3^{j}-1,4,17\right],\left[3^{j+1}, 5,19\right]\right), \\
& \left(\left[2 \cdot 3^{j}-1,4,17\right],\left[3^{j+1}, 5,19\right]\right), \quad \text { for } 1 \leq j \leq 22, \\
& \left(\left[3^{23}-1,4,17, j\right],\left[3^{j}, 5,19,23\right]\right), \quad \text { for } 1 \leq j \leq 23 ;
\end{aligned}
$$

those congruent to 18 modulo 19 by

$$
\begin{gathered}
\left(\left[3^{j}-1,7,18\right],\left[3^{j+1}, 7,19\right]\right), \\
\left(\left[2 \cdot 3^{j}-1,7,18\right],\left[3^{j+1}, 7,19\right]\right), \quad \text { for } 1 \leq j \leq 22, \\
\left(\left[3^{23}-1,7,18, j\right],\left[3^{j}, 7,19,23\right]\right), \quad \text { for } 1 \leq j \leq 23 ;
\end{gathered}
$$

those congruent to 19 (or 0) modulo 19 by

$$
\begin{aligned}
& \left(\left[3^{j}-1,4,7,19\right],\left[3^{j+1}, 5,7,19\right]\right), \\
& \left(\left[2 \cdot 3^{j}-1,4,7,19\right],\left[3^{j+1}, 5,7,19\right]\right), \quad \text { for } 1 \leq j \leq 22, \\
& \left(\left[3^{23}-1,4,7,19, j\right],\left[3^{j}, 5,7,19,23\right]\right), \quad \text { for } 1 \leq j \leq 23 .
\end{aligned}
$$

The congruences above combine then to cover $\mathcal{I}([2],[3])$.

Next, we use an approach similar to the case of $\mathcal{I}([2],[3])$ and break up $\mathcal{I}([3],[3])$ into the five residue classes modulo 5. The second congruence in $(3.1)$ will cover $\mathcal{I}([3,1],[3,5])$. In each of the four remaining cases $\mathcal{I}([3, j],[3,5])$, with $2 \leq j \leq 5$, we will divide the integers up into their residue classes modulo 7 . What is of particular importance to us here is that the first congruence in (3.4) and the congruences

$$
([3,2],[3,7]), \quad([3,3],[3,7])
$$


cover three of the seven residue classes modulo 7 for each $\mathcal{I}([3, j],[3,5])$. Further, we can cover a fourth residue class modulo 7 in each $\mathcal{I}([3, j],[3,5])$ by using the congruences

$$
\begin{gathered}
\left(\left[3^{j}, 5\right],\left[3^{j+1}, 7\right]\right), \quad\left(\left[2 \cdot 3^{j}, 5\right],\left[3^{j+1}, 7\right]\right), \quad \text { for } 1 \leq j \leq 22, \\
\left(\left[3^{23}, 5, j\right],\left[3^{j}, 7,23\right]\right), \quad \text { for } 1 \leq j \leq 23 .
\end{gathered}
$$

To finish covering $\mathcal{I}([3],[3])$, we are left with covering the integers congruent to 4,6 and 7 modulo 7 in each $\mathcal{I}([3, j],[3,5])$, with $2 \leq j \leq 5$. We note that we have deliberately covered the residue class 5 modulo 7 instead of 4 modulo 7 so that we can make use of the last congruence in (3.4) when we consider $\mathcal{I}([3,4],[3,5])$.

We finish covering $\mathcal{I}([3,2],[3,5])$ as follows. As noted above, the congruences in (3.14) and (3.15) cover four residue classes modulo 7. We make use of this momentarily, but for the time being we instead break up the integers in $\mathcal{I}([3,2],[3,5])$ into their five residue classes modulo $5^{2}$ with the goal of covering each of these five classes in turn. The congruences corresponding to $j=1$ in the list

$$
\left(\left[3,5^{j}+2\right],\left[3,5^{j+1}\right]\right), \quad\left(\left[3,2 \cdot 5^{j}+2\right],\left[3,5^{j+1}\right]\right), \quad \text { for } 1 \leq j \leq 22,
$$

cover the residue classes 7 and 12 modulo $5^{2}$. The collection of $2 \cdot 22+23$ congruences corresponding to $j=1$ in

$$
\begin{aligned}
& \left(\left[3^{i}, 3 \cdot 5^{j}+2\right],\left[3^{i+1}, 5^{j+1}\right]\right), \\
& \left(\left[2 \cdot 3^{i}, 3 \cdot 5^{j}+2\right],\left[3^{i+1}, 5^{j+1}\right]\right), \quad \text { for } 1 \leq i, j \leq 22, \\
& \left(\left[3^{23}, 3 \cdot 5^{j}+2, i\right],\left[3^{i}, 5^{j+1}, 23\right]\right), \quad \text { for } 1 \leq i \leq 23,1 \leq j \leq 22
\end{aligned}
$$

cover the integers that are 17 modulo $5^{2}$. To cover the integers that are 22 modulo $5^{2}$, we consider their residue classes modulo 7 and recall that we have already covered the integers in $\mathcal{I}([3,2],[3,5])$ that are $1,2,3$ and 5 modulo 7 . We use the case $j=1$ in

$$
\begin{aligned}
&\left(\left[4 \cdot 5^{j}+2,4\right],\left[5^{j+1}, 7\right]\right), \quad\left(\left[4 \cdot 5^{j}+2,6\right],\left[5^{j+1}, 7\right]\right) \\
&\left(\left[3,4 \cdot 5^{j}+2,7\right],\left[3,5^{j+1}, 7\right]\right), \quad \text { for } 1 \leq j \leq 23,
\end{aligned}
$$

to finish covering the integers that are 22 modulo $5^{2}$. We still need to cover $\mathcal{I}\left([3,2],\left[3,5^{2}\right]\right)$. We divide these into five residue classes modulo $5^{3}$ and use $j=2$ in (3.16), (3.17) and (3.18) to cover the four of these five classes that are not 2 modulo $5^{3}$. Continuing with $3 \leq j \leq 22$ to cover residue classes modulo $5^{j+1}$, we see that the congruences in (3.16), (3.17) and (3.18) cover all the integers in $\mathcal{I}\left([3,2],\left[3,5^{2}\right]\right)$ except those that are in $\mathcal{I}\left([3,2],\left[3,5^{23}\right]\right)$. We cover these by using the congruences

$$
\left([2, j],\left[5^{j}, 23\right]\right), \quad \text { for } 1 \leq j \leq 23,
$$


noting that the $j$ th congruence in this list covers those integers in $\mathcal{I}\left([3,2],\left[3,5^{23}\right]\right)$ that are $j$ modulo 23 .

Next, we cover $\mathcal{I}([3,3],[3,5])$. We break up these integers into their residue classes modulo 7 . Recall we only need to cover the residue classes modulo 4, 6 and 7 modulo 7. In (3.4), the modulus $5 \cdot 7$ was used once, and we use it again here to cover $\mathcal{I}([3,3,4],[3,5,7])$ with

$$
([3,4],[5,7]) \text {. }
$$

We cover all integers in $\mathcal{I}([3,3,6],[3,5,7])$ except those congruent to 6 modulo 11 using

$$
\begin{gathered}
([1],[11]), \quad([3,2],[3,11]), \quad([3,3],[3,11]), \quad([3,4],[5,11]), \\
([3,3,5],[3,5,11]), \quad([3,6,7],[3,7,11]), \quad([3,6,8],[3,7,11]), \\
([6,9],[7,11]), \quad([6,10],[7,11]), \quad([3,6,11],[5,7,11]) .
\end{gathered}
$$

For later purposes, note that we have only used some moduli dividing $3 \cdot 5$. $7 \cdot 11$ above once. To cover $\mathcal{I}([3,3,6,6],[3,5,7,11])$, we use

$$
\begin{gathered}
\left(\left[3^{j}, 6\right],\left[3^{j+1}, 11\right]\right),\left(\left[2 \cdot 3^{j}, 6\right],\left[3^{j+1}, 11\right]\right), \quad \text { for } 1 \leq j \leq 22, \\
\left(\left[3^{23}, 6, j\right],\left[3^{j}, 11,23\right]\right), \quad \text { for } 1 \leq j \leq 23 .
\end{gathered}
$$

Next, we turn to $\mathcal{I}([3,3,7],[3,5,7])$ and consider their residue classes modulo 13. We cover all integers in $\mathcal{I}([3,3,7],[3,5,7])$ except those congruent to 12 and 13 modulo 13 in a manner very similar to our approach for covering $\mathcal{I}([3,3,6],[3,5,7])$ above but with 11 replaced by 13 . Specifically, we use

$$
\begin{gathered}
([1],[13]), \quad([3,2],[3,13]), \quad([3,3],[3,13]), \quad([3,4],[5,13]), \\
([3,3,5],[3,5,13]), \quad([3,7,7],[3,7,13]), \quad([3,7,8],[3,7,13]), \\
([7,9],[7,13]), \quad([7,10],[7,13]), \quad([3,7,11],[5,7,13]) .
\end{gathered}
$$

and

$$
\begin{gathered}
\left(\left[3^{j}, 6\right],\left[3^{j+1}, 13\right]\right),\left(\left[2 \cdot 3^{j}, 6\right],\left[3^{j+1}, 13\right]\right), \quad \text { for } 1 \leq j \leq 22, \\
\left(\left[3^{23}, 6, j\right],\left[3^{j}, 13,23\right]\right), \quad \text { for } 1 \leq j \leq 23 .
\end{gathered}
$$

We use

$$
([3,3,7,12],[3,5,7,13])
$$

to cover $\mathcal{I}([3,3,7,12],[3,5,7,13])$ and

$$
\begin{gathered}
\left(\left[3^{j}, 7,13\right],\left[3^{j+1}, 7,13\right]\right),\left(\left[2 \cdot 3^{j}, 7,13\right],\left[3^{j+1}, 7,13\right]\right), \text { for } 1 \leq j \leq 22, \\
\left(\left[3^{23}, 7,13, j\right],\left[3^{j}, 7,13,23\right]\right), \quad \text { for } 1 \leq j \leq 23 .
\end{gathered}
$$

to cover $\mathcal{I}([3,3,7,13],[3,5,7,13])$. We deduce that the congruences $(3.20)$ (3.26) cover $\mathcal{I}([3,3],[3,5])$. 
To cover $\mathcal{I}([3,4],[3,5])$, we need only cover those integers that are in the residue classes modulo 4, 6 and 7 modulo 7 (the other residue classes being covered already above). The congruence $([4,4],[5,7])$ in $(3.4)$ covers the integers in $\mathcal{I}([3,4,4],[3,5,7])$. To cover $\mathcal{I}([3,4,6],[3,5,7])$, we can reuse several of the congruences in (3.21) and (3.22) (those with moduli not divisible by 5$)$ to cover the integers in certain residue classes modulo 11 . Specifically, the integers in $\mathcal{I}([3,4,6],[3,5,7])$ that are $1,2,3,6,7,8,9$ or 10 modulo 11 are covered by congruences in (3.21) and (3.22). We only used the moduli $5 \cdot 11,3 \cdot 5 \cdot 11$ and $5 \cdot 7 \cdot 11$ once in $(3.21)$, so we use now

$$
([4,4],[5,11]), \quad([3,4,5],[3,5,11]), \quad([4,6,11],[5,7,11])
$$

to cover the integers in $\mathcal{I}([3,4,6],[3,5,7])$ that are 4,5 or 11 modulo 11. This completes covering $\mathcal{I}([3,4,6],[3,5,7])$. Turning to $\mathcal{I}([3,4,7],[3,5,7])$, we can reuse congruences in (3.23), (3.24) and (3.26) to cover those integers here that lie in the residue classes $1,2,3,6,7,8,9,10$ or 13 modulo 13 . We use

$$
\begin{array}{cc}
([4,4],[5,13]), & ([3,4,5],[3,5,13]), \\
([4,7,11],[5,7,13]), & ([3,4,7,12],[3,5,7,13])
\end{array}
$$

to cover the remaining integers in $\mathcal{I}([3,4,7],[3,5,7])$.

We are left with covering $\mathcal{I}([3,5],[3,5])$. More precisely, we need only cover $\mathcal{I}([3,5,4],[3,5,7]), \mathcal{I}([3,5,6],[3,5,7])$ and $\mathcal{I}([3,5,7],[3,5,7])$. We split up the integers in $\mathcal{I}([3,5,4],[3,5,7])$ into residue classes modulo 17 . Let $a \in \mathcal{I}([3,5,4],[3,5,7])$, and let $m_{1}, m_{2}, \ldots, m_{7}$ be the divisors of $3 \cdot 5 \cdot 7$ that are $>1$. We use

$$
\left([a, j],\left[m_{j}, 17\right]\right), \quad\left([a, j+7],\left[m_{j}, 17\right]\right), \quad \text { for } 1 \leq j \leq 7,
$$

to cover the integers in $\mathcal{I}([3,5,4],[3,5,7])$ that are in the residue classes $1,2,3, \ldots, 13$ or 14 modulo 17 . We cover the remaining integers using

$$
\begin{gathered}
\left(\left[3^{j}, 16\right],\left[3^{j+1}, 17\right]\right),\left(\left[2 \cdot 3^{j}, 16\right],\left[3^{j+1}, 17\right]\right), \quad \text { for } 1 \leq j \leq 22, \\
\left(\left[3^{23}, 16, j\right],\left[3^{j}, 17,23\right]\right), \quad \text { for } 1 \leq j \leq 23
\end{gathered}
$$

and

$$
\begin{gathered}
\left(\left[3^{j}, 5,17\right],\left[3^{j+1}, 5,17\right]\right),\left(\left[2 \cdot 3^{j}, 5,17\right],\left[3^{j+1}, 5,17\right]\right), \text { for } 1 \leq j \leq 22, \\
\left(\left[3^{23}, 5,17, j\right],\left[3^{j}, 5,17,23\right]\right), \quad \text { for } 1 \leq j \leq 23 .
\end{gathered}
$$

We turn to covering $\mathcal{I}([3,5,6],[3,5,7])$ and once again use the congruences in $(3.21)$ and (3.22). With these, we cover the integers in $\mathcal{I}([3,5,6],[3,5,7])$ that are $1,2,3,6,7,8,9$ or 10 modulo 11 . We use

$$
([3,5,6,4],[3,5,7,11]), \quad([3,5,6,5],[3,5,7,11]),
$$


to cover those integers in $\mathcal{I}([3,5,6],[3,5,7])$ that are 4 or 5 modulo 11 . We cover those that are 11 modulo 11 using

$$
\begin{gathered}
\left(\left[3^{j}, 5,11\right],\left[3^{j+1}, 5,11\right]\right),\left(\left[2 \cdot 3^{j}, 5,11\right],\left[3^{j+1}, 5,11\right]\right), \text { for } 1 \leq j \leq 22, \\
\left(\left[3^{23}, 5,11, j\right],\left[3^{j}, 5,11,23\right]\right), \quad \text { for } 1 \leq j \leq 23 .
\end{gathered}
$$

For $\mathcal{I}([3,5,7],[3,5,7])$, we use congruences in $(3.23),(3.24)$ and (3.26) again to cover integers in the residue classes $1,2,3,6,7,8,9,10$ or 13 modulo 13. The congruences

$$
\begin{gathered}
\left(\left[3^{j}, 5,4\right],\left[3^{j+1}, 5,13\right]\right),\left(\left[2 \cdot 3^{j}, 5,4\right],\left[3^{j+1}, 5,13\right]\right), \quad \text { for } 1 \leq j \leq 22, \\
\left(\left[3^{23}, 5,4, j\right],\left[3^{j}, 5,13,23\right]\right), \quad \text { for } 1 \leq j \leq 23 .
\end{gathered}
$$

and

$$
\begin{gathered}
\left(\left[3^{j}, 5,7,5\right],\left[3^{j+1}, 5,7,13\right]\right), \\
\left(\left[2 \cdot 3^{j}, 5,7,5\right],\left[3^{j+1}, 5,7,13\right]\right), \quad \text { for } 1 \leq j \leq 22, \\
\left(\left[3^{23}, 5,7,5, j\right],\left[3^{j}, 5,7,13,23\right]\right), \quad \text { for } 1 \leq j \leq 23 .
\end{gathered}
$$

cover the integers in $\mathcal{I}([3,5,7],[3,5,7])$ that are in the residue classes 4 or 5 modulo 13. We can use the congruences in (3.21) and (3.22) to cover those integers in each of $\mathcal{I}([3,5,7,11],[3,5,7,13])$ and $\mathcal{I}([3,5,7,12],[3,5,7,13])$ that are $1,2,3$ or 6 modulo 11 . Recall $m_{1}, m_{2}, \ldots, m_{7}$ are the divisors of $3 \cdot 5 \cdot 7$ that are $>1$. Let $b \in \mathcal{I}([3,5,7],[3,5,7])$. We cover the remaining integers using

$$
\begin{aligned}
([b, j+3,11], & {\left.\left[m_{j}, 11,13\right]\right), } \\
& \left([b, j+3,12],\left[m_{j}, 11,13\right]\right), \text { for } 1 \leq j \leq 2,
\end{aligned}
$$

and

$$
\begin{aligned}
([b, j+4,11], & {\left.\left[m_{j}, 11,13\right]\right), } \\
& \left([b, j+4,12],\left[m_{j}, 11,13\right]\right), \text { for } 3 \leq j \leq 7 .
\end{aligned}
$$

We note that the modulus $11 \cdot 13$, which could have been used twice, was not used here.

As just shown, the congruences in (3.1) - (3.38) form a covering of the integers. We use these congruences to form the set $\mathcal{S}$ needed for Lemma 2.2. What is left is to verify the conditions (i), (ii) and (iii) in Lemma 2.2, which can be done directly going through the various moduli indicated above. This completes the proof.

\section{Concluding Remarks}

We made use of 2773 congruences for the construction given in the previous section, that is to obtain a covering satisfying the conditions in Lemma 2.2. 
This corresponds to 5539 congruences to construct a polynomial $f(x) \in$ $\mathbb{Z}[x]$, based on Lemma 2.1 , such that $f(1) \neq-2$ and $f(x) x^{n}+2$ is reducible for all integers $n \geq 0$. Although the method used in [1] is similar to the approach here, the covering system obtained for constructing an analogous $f(x)$ with $d=4$ there was more complicated due to the fact that prime moduli were not used (i.e., Lemma 2.2 (ii) was not considered in [1]).

The approach given by L. Jones in [2] was to show first that there is an $f(x) \in \mathbb{Z}[x]$ such that $f(1) \neq-d$ and $f(x) x^{n}+d$ is reducible for all integers $n \geq 0$ if there is a covering system with distinct moduli $>1$ and with the least common multiple of the moduli equal to $d$. That such an $f(x)$ exists in this case would also follow from Lemma 1 in Section 2 which originally appeared in [1]. To see this, one should place all the moduli which are powers of 2 besides 2 itself in the collection of congruences given in (2.2) and then either add a congruence or translate so that the congruence in (2.1) is used with $k=1$. L. Jones then uses this information to show that an $f(x)$ can be constructed as above for infinitely many $d \equiv 2 \bmod 4$. In particular, he is able to produce an explicit $f(x)$ in the case $d=90$.

Acknowledgment: The first author gratefully acknowledges the support of the NSA during the writing of this paper.

\section{References}

[1] M. Filaseta, Coverings of the integers associated with an irreducibility theorem of A. Schinzel, in Number theory for the millennium, II (Urbana, IL, 2000), pages 1-24, A K Peters, Natick, MA, 2002.

[2] L. Jones, Polynomial variations on a theme of Sierpiński, Int. J. Number Theory 5 (2009), 999-1015.

[3] A. Schinzel, Reducibility of polynomials and covering systems of congruences, Acta. Arith. 13 (1967), 91-101.

[4] W. Sierpiński, Sur un problème concernant les nombres $k \cdot 2^{n}+1$, Elem. Math. 15 (1960), 73-74. 